\title{
Molecular basis for extender unit specificity of mycobacterial polyketide synthases
}

Anna D. Grabowska, ${ }^{1,2 \#}$ Yoann Brison, ${ }^{1,3 \#}$ Laurent Maveyraud, ${ }^{1 \#}$ Sabine Gavalda, ${ }^{1,4}$ Alexandre Faille, ${ }^{1,5}$ Virginie Nahoum, ${ }^{1}$ Cécile Bon, ${ }^{1}$ Christophe Guilhot,${ }^{1}$ Jean-Denis Pedelacq, ${ }^{1 *}$ Christian Chalut, ${ }^{1 *}$ and Lionel Mourey ${ }^{1 *}$

${ }^{1}$ Institut de Pharmacologie et de Biologie Structurale, IPBS, Université de Toulouse, CNRS, UPS, 31077 Toulouse, France

${ }^{2}$ Present address: Department of Biophysics and Human Physiology, Faculty of Health Sciences, Medical University of Warsaw, Poland

${ }^{3}$ Present address: Toulouse White Biotechnology, TWB, INRAE, INSA, CNRS, 31520 Ramonville Saint-Agne, France

${ }^{4}$ Present address: Toulouse Biotechnology Institute, TBI, Université de Toulouse, CNRS, INRAE, INSA, Toulouse, France

${ }^{5}$ Present address: Cambridge Institute for Medical Research, University of Cambridge, Cambridge CB2 0XY, UK

*Corresponding author: jean-denis.pedelacq@ipbs.fr, christian.chalut@ipbs.fr, lionel.mourey@ipbs.fr

"Equal contribution

\section{Table of Contents}

S1-S4 Methods

S5-S6 Supporting Information Figure Legends

S7 Supporting Information Table 1. Crystallographic data collection and refinement statistics

S8 Supporting Information Table 2. PDB AT structures that include a L linker domain

S9-S10 Supporting Information References

S11 Supporting Information Figure 1. Schematic representation of the reactions catalyzed by the mycobacterial PKSs studied in this work

S12 Supporting Information Figure 2. SAXS analysis of molecular species containing the AT domain of PpsA.

S13 Supporting Information Figure 3. Structure-based multiple sequence alignment of LAT-Pks13, L-AT-Mas, AT-PpsA, and AT-PpsC

S14 Supporting Information Figure 4. Topography of the active sites

S15 Supporting Information Figure 5. Ligand-binding channels

S16 Supporting Information Figure 6. Zoom-in on extender unit specificity for wild type L-AT-Mas and mutant proteins 


\section{METHODS}

Cloning. Construction of the vector pETMas, which enables expression of the recombinant full-length Mas protein from M. tuberculosis H37Rv fused to a poly-His tag at its C-terminus has been described elsewhere. ${ }^{l}$ The $p p s A$ and $p p s C$ genes were amplified by PCR from M. bovis BCG Pasteur 1173P2 genomic DNA and inserted into a pET28a vector (Novagen) downstream from the T7 promoter, to yield pETA and pETC, respectively. DNA fragments encoding the AT domains of Mas (residues P447-W871) and PpsA (residues V542-P959 or N621-H952) were amplified from pETMas and pETA, respectively. In-frame fragments centered onto the AT domain of PpsC were obtained from a large-size DNA library (850-1650 bp) created from the PCR-amplified $p p s C$, as previously described. ${ }^{2,3}$ The bipartite split-GFP system GFP110/GFP11 was then used to identify the most soluble constructs. ${ }^{4,5}$ All AT domains were inserted into a pET28a vector (Novagen) downstream from the T7 promoter. These vectors allow the production of the recombinant AT domains with a poly-His tag fused to their Nterminus. Plasmids expressing variants of the AT-Mas domains were generated by site-directed mutagenesis using the QuikChange II XL kit (Stratagene) according to manufacturer's instructions. The resulting plasmids were analyzed by DNA sequencing. The amino acid sequences of the AT domain of Mas, PpsA and PpsC domains are well conserved between $M$. bovis and M. tuberculosis strains, displaying high degree of identity, i.e. 100\% for AT-Mas, 99\% for PpsA and 100\% for AT-PpsC.

Production and purification of recombinant proteins. For crystallographic studies, the recombinant AT domains of PpsA and $\mathrm{PpsC}$, and the wild-type and mutant AT-Mas proteins were expressed in Escherichia coli BL21 (DE3). Cultures were carried out in LB broth. Heterologous protein expression was induced by adding $0.1-0.5 \mathrm{mM}$ of IPTG when OD600 reached $\sim 0.5$. Cells were allowed to grow for $4 \mathrm{~h}$ at $23{ }^{\circ} \mathrm{C}$ or $30{ }^{\circ} \mathrm{C}$. The pelleted cells were suspended in $40 \mathrm{ml}$ of buffer A (100 mM Tris- $\mathrm{HCl}, 150 \mathrm{mM} \mathrm{NaCl}, \mathrm{pH} 8.0)$ and lysed by sonication on ice prior to centrifugation at $30,000-40,000 \times g$ for $30 \mathrm{~min}$. The supernatants were poured on $2-3 \mathrm{ml}$ of cobalt Talon Superflow resin (Clontech) previously washed with buffer A. The resin was then washed 3 times with buffer A before the elution of non-specific contaminants with buffer A containing $10 \mathrm{mM}$ imidazole and finally the elution of the bound protein with buffer A containing $250 \mathrm{mM}$ imidazole. The eluted proteins were concentrated using a Vivaspin 20 centrifugal concentrator (Sartorius), prior to injection into a HiLoad 16/60 Superdex 75 (GE Healthcare) pre-equilibrated with 25-100 mM Tris-HCl, 25-150 mM NaCl, $\mathrm{pH}$ 8.0. Freshly purified enzymes were concentrated and screened for crystallization conditions. 
For the wild-type and mutant AT-Mas proteins used in the ligand binding and competitive binding assays, expression plasmids were transformed into E. coli strain BL21(DE3)pLysS (Novagen) and proteins were expressed in the same conditions as above at $30{ }^{\circ} \mathrm{C}$. For each recombinant protein, cell pellets corresponding to $100 \mathrm{ml}$ of culture were resuspended in $10 \mathrm{ml}$ of lysis buffer (buffer A supplemented with $10 \mathrm{mM}$ imidazole and $1 \mathrm{mM}$ phenylmethylsulfonyl fluoride (PMSF), and sonicated on ice. The lysate was centrifuged at 22,000 $\times g$ for $30 \mathrm{~min}$ and the supernatant was applied to a 1-ml HisTrap ${ }^{\mathrm{TM}} \mathrm{HP}$ column (GE Healthcare) equilibrated with buffer A containing $10 \mathrm{mM}$ imidazole and $0.2 \mathrm{mM}$ PMSF. After extensive washing with buffer A containing successively 10,20 and $50 \mathrm{mM}$ imidazole, recombinant proteins were eluted with buffer A supplemented with $150 \mathrm{mM}$ imidazole. Fractions containing the highest concentrations of recombinant proteins were pooled and loaded onto a HiLoad 16/60 Superdex 75 equilibrated with buffer $\mathrm{A}$ and $0.2 \mathrm{mM}$ PMSF. Fractions containing soluble monomeric proteins were pooled, concentrated by centrifugation, and used for further experiments or stored at $-20{ }^{\circ} \mathrm{C}$ in the presence of $50 \%(\mathrm{v} / \mathrm{v})$ glycerol. To produce the wildtype and the mutant forms of the full-length Mas protein, expression vectors were introduced into an E. coli BL21(DE3) $\Delta e n t D$ strain harboring the $\mathrm{pLSfp}$ vector, which encodes the surfactin phosphopantetheinyl transferase (Sfp) from Bacillus subtilis, to allow posttranslational modification of the acyl carrier protein (ACP) domain of Mas ${ }^{1,6}$. Proteins were expressed and purified as described above with the exception that size exclusion chromatography was performed using a HiLoad 16/60 Superdex 200. Fractions containing recombinant soluble monomeric proteins were pooled, concentrated by ultrafiltration, and used for further experiments or stored at $-20{ }^{\circ} \mathrm{C}$ in the presence of $50 \%(\mathrm{v} / \mathrm{v})$ glycerol.

Ligand binding and competitive binding assays. For ligand binding assays, $5 \mu \mathrm{M}$ of AT domain or $1 \mu \mathrm{M}$ of Mas full-length protein were incubated with $50 \mu \mathrm{M}$ of $\left[2-{ }^{14} \mathrm{C}\right] \mathrm{MM}-\mathrm{CoA}$ $\left(\mathrm{ARC}, 55 \mathrm{Ci} \mathrm{mol}^{-1}\right)$ or $\left[2{ }^{14} \mathrm{C}\right] \mathrm{M}-\mathrm{CoA}\left(\mathrm{ARC}, 55 \mathrm{Ci} \mathrm{mol}^{-1}\right)$ in $100 \mathrm{mM}$ Tris- $\mathrm{HCl}, \mathrm{pH} 7.0$ for 1 h at $30{ }^{\circ} \mathrm{C}$ (or exceptionally overnight incubation at $4{ }^{\circ} \mathrm{C}$ for AT-Mas:M624V-S726F variant and Mas:M624V-S726F variant). ${ }^{6}$ The samples were separated by SDS-PAGE and analyzed by phosphorimaging (Variable Mode Imager Typhoon TRIO, Amersham Biosciences) to quantitate radiolabeled proteins and Coomassie Blue staining. All binding assays were performed at least in triplicate. Competitive binding assays were performed using identical experimental procedures. Proteins were incubated with $50 \mu \mathrm{M}$ of radiolabeled MM-CoA or M$\mathrm{CoA}$, in the absence or in the presence of increasing concentrations $(25,50$ and $100 \mu \mathrm{M})$ of non-radiolabeled substrates. All competitive assays were performed at least in duplicate. 
Small-angle X-ray scattering experiments (SAXS). For SAXS experiments, the purification of AT-PpsA was adapted as follows. For the 542-959 construct, the concentrated enzyme obtained after affinity chromatography was injected onto a HiLoad 16/60 Superdex 75 (GE Healthcare) pre-equilibrated with $50 \mathrm{mM}$ Tris- $\mathrm{HCl}, 300 \mathrm{mM} \mathrm{NaCl}, \mathrm{pH}$ 8.0. For the shorter form of AT-PpsA (residues 621-952), the size exclusion chromatography buffer composition was 25 $\mathrm{mM}$ Tris- $\mathrm{HCl}, 250 \mathrm{mM} \mathrm{NaCl}, \mathrm{pH}$ 7.5. The elution peaks were concentrated using a Vivaspin 20 centrifugal concentrator (Sartorius) to a concentration of $10 \mathrm{mg} \mathrm{mL}^{-1}$. SAXS experiments were carried out in HPLC mode on the SWING beamline at the SOLEIL synchrotron, Gif-surYvette, France. $40 \mu \mathrm{L}$ of concentrated AT-PpsA were injected onto a Bio SEC-3 (300 ̊ pore size) HPLC column (Agilent) pre-equilibrated with the purification buffer at a flow rate of 0.15 $\mathrm{mL} \mathrm{min}-1$ and a temperature of $15^{\circ} \mathrm{C}$. Data were collected in a $Q$ range of $0.06-6.01 \mathrm{~nm}^{-1}$ and $0.05-4.35 \mathrm{~nm}^{-1}$ for the longest and shortest forms, respectively, with $Q=4 \pi \sin \theta / \lambda$ and a scattering angle of $2 \theta$. A total of 249 frames of $1.5 \mathrm{~s}$ each were collected throughout the whole elution time. Data were reduced with the in-house FOXTROT application. After buffer subtraction, Guinier analysis at low $Q$ was performed on each curve to check for the stability of the associated radius of gyration $\left(R_{\mathrm{g}}\right)$, and the selected frames were averaged. Data were then analyzed using the ATSAS suite. ${ }^{7}$

Crystallization and structure determination. Purified wild type or L-AT-Mas mutant proteins and AT-PpsA were concentrated to $6.5 \mathrm{mg} \mathrm{mL}^{-1}$ and $9.5 \mathrm{mg} \mathrm{mL}^{-1}$, respectively. All L-AT-Mas proteins crystallized at $20{ }^{\circ} \mathrm{C}$ in $1.1 \mathrm{M}$ sodium succinate, $0.1 \mathrm{M}$ sodium acetate buffer at an initial $\mathrm{pH}$ of 4.2. AT-PpsA crystallized at $12{ }^{\circ} \mathrm{C}$ in $19 \%(\mathrm{w} / \mathrm{w})$ PEG 5,000 MME, 0.1 M MES pH 6.5. AT-PpsC, concentrated to $8.4 \mathrm{mg} \mathrm{mL}^{-1}$, crystallized at $12{ }^{\circ} \mathrm{C}$ in $1.8 \mathrm{M}$ ammonium sulfate, $0.2 \mathrm{M}$ sodium thiocyanate, $0.1 \mathrm{M}$ Tris- $\mathrm{HCl} \mathrm{pH}$ 8.0. The volume ratio was 1:1 $\mu \mathrm{L}$ between the purified enzyme solutions and crystallization solutions. L-AT-Mas crystals were cryo-cooled in a gaseous flux of nitrogen at $100 \mathrm{~K}$ using a cryoprotectant solution composed of the reservoir solution supplemented with $10 \%(\mathrm{v} / \mathrm{v})$ ethylene glycol or glycerol. AT-PpsA and AT-PpsC crystals were cryoprotected with the reservoir solution supplemented with $15 \%$ (v/v) glycerol. The best method for getting L-AT-Mas complexes with M-CoA or MM-CoA (Sigma-Aldrich) was the direct addition in the hanging drops containing the crystals of $0.2 \mu \mathrm{L}$ of a $50 \mathrm{mM}$ ligand solution prepared with the reservoir solution (final concentration of $4.5 \mathrm{mM}$ ) for $2 \mathrm{~h}$ prior to flash-freezing. Datasets were collected either at the European Synchrotron Radiation Facility (ESRF, Grenoble, France) or at the SOLEIL synchrotron (Gifsur-Yvette, France). All data were indexed, integrated and scaled using $\mathrm{XDS}^{8}$ or programs from 
the CCP4 software suite, which were also used for crystallographic calculations. ${ }^{9,}{ }^{10}$ All wild type structures were solved by molecular replacement using PHASER ${ }^{11}$ and either the X-ray structure of L-AT-Pks13 (PDB code 3TZW) in the case of L-AT-Mas and AT-PpsA or that of the AT domain from DEBS module 3 (PDB code 2QO3) for AT-PpsC. Iterative cycles of manual model building in $\mathrm{COOT}^{12}$ and refinement procedures using PHENIX refine ${ }^{13}$, REFMAC $^{10}$ and BUSTER ${ }^{14}$ were applied until convergence. Details of data collection, cell parameters, processing and refinement statistics are presented in Table S1. Protein structure databases were searched and structures were superimposed with Dali software ${ }^{15,} 16$ and SUPERPOSE from the CCP4 software package. Secondary structure assignment was performed using DSSP. ${ }^{17,18}$ All structures and the electrostatic potential of protein surfaces were visualized with PyMOL. ${ }^{19}$ The sequence alignment was generated with ESPript $3 .^{20}$

\section{Accession Codes}

The atomic coordinates and crystallographic structure factors of proteins and complexes described in this work have been deposited in the Protein Data Bank (www.rcsb.org) with accession codes as follows: AT-PpsA, 7AKC; AT-PpsC, 7AHB; L-AT-Mas, 7AGP; methylmalonylated L-AT-Mas, 7AGS; S726F L-AT Mas, 7AGQ; methylmalonylated S726F L-AT-Mas, 7AGU; malonylated S726F L-AT-Mas, 7AGT; M624V-S726F L-AT-Mas soaked with MM-CoA, 7AGR. 


\section{SUPPORTING INFORMATION FIGURE LEGENDS}

Supporting Information Figure 1. Schematic representation of the reactions catalyzed by the mycobacterial PKSs studied in this work. (a) Pks13 is involved in the condensation reaction between two activated long chain fatty acids, a meromycoloyl-AMP and a carboxy-acyl-CoA, and the transfer of the product on an acceptor, e.g. trehalose leading to trehalose monomycolate precursors. (b) PpsA and PpsC are M-CoA-dependent monomodular PKSs involved, together with PpsB, PpsD and PpsE, in the elongation of long-chain fatty acid backbones to form (phenol)phthiocerol chains. (c) Mas is an iterative PKS that uses MM-CoA to produce methylbranched mycocerosates, which are then esterified to the two hydroxyl groups of the (phenol)phtiocerol chain to form DIMs and PGLs. $\mathrm{R}_{1}=$ meromycolic chain of variable size and structure $;{ }^{21} \mathrm{R}_{2}=\mathrm{CH}_{3}-\left(\mathrm{CH}_{2}\right)_{21-23} ; \mathrm{R}_{3}(\mathrm{DIMS})=\mathrm{CH}_{3}-\left(\mathrm{CH}_{2}\right)_{20-22} ; \mathrm{R}_{4}(\mathrm{DIMS})=\mathrm{CH}_{3}-\left(\mathrm{CH}_{2}\right)_{16}-$ 18.

Supporting Information Figure 2. SAXS analysis of molecular species containing the AT domain of PpsA. (a) Experimental data. (b) Kratky plots. Curves corresponding to the longest (residues 542-959) and shortest (residues 621-952) constructs are in blue and red, respectively. The shortest construct, which included only the core AT domain of PpsA displayed less flexibility and allowed to obtain well-diffracting crystals.

Supporting Information Figure 3. Structure-based multiple sequence alignment of L-ATPks13, L-AT-Mas, AT-PpsA, and AT-PpsC. The sequence numbering is for L-AT-Pks13. Sequence homology is highlighted in red, whereas sequence identity is shown as white letters on a red background. Structurally aligned and unaligned residues are displayed in uppercase and lowercase, respectively, taking L-AT-Pks13 as reference. Residues absent from the structures are on a green background. Secondary structure elements as deduced from the corresponding crystal structures are indicated. Those at the top are for L-AT-Pks13 and were colored according to the different subdomains depicted in Figure 2. The catalytic residues are indicated by triangles, the arginine residue involved in selecting carboxylated substrates is indicated by a star.

Supporting Information Figure 4. Topography of the active sites. (a) Molecular surface. Residues of L-AT-Pks13 delineating the groove overhanging the active site and the exit of the channel are colored in light gray and highlighted in black. Corresponding areas of L-AT-Mas, AT-PpsA and AT-PpsC are also shown. (b) Semi-perpendicular view. 
Supporting Information Figure 5. Ligand-binding channels. (a) The carboxypalmitoyl group in its dedicated tunnels in L-AT-Pks13 (PDB code 3TZZ). Residues delineating the tunnels are represented as sticks and labeled. Atoms found within $5 \AA$ of the ligand are shown as enlarged sticks. Protein (respectively ligand) atoms found within $3.5 \AA$ distances of ligand (respectively protein) atoms are colored violet. The two conformations (Conf 1 and Conf2) of the carboxypalmitoyl group and their chemical environment are depicted. (b) Corresponding area in L-AT-MAS. (c) AT-PpsA. (d) AT-PpsC. Residues blocking the tunnels are shown and labeled in red.

Supporting Information Figure 6. Zoom-in on extender unit specificity for wild type L-ATMas and mutant proteins. (a) Structure of (2S)-methylmalonylated wild type L-AT-Mas. (b) Structure of (2S)-methylmalonylated S726F L-AT-Mas. (c) Structure of malonylated S726F LAT-Mas. (d) Structure of unbound L-AT-Mas M624V-S726F. Structures are shown as black ribbon and were superimposed with native L-AT-Mas (color-coded as in Figure 2). The ligands are in green and the mutated residues in red. The orientation is the same as in Figure 6. Featureenhanced map $(\mathrm{FEM})^{22}$ contoured at $1.0 \sigma$ is shown in blue for residues from the active site. 
Supporting Information Table 1. Crystallographic data collection and refinement statistics.

\begin{tabular}{|c|c|c|c|c|c|c|c|c|}
\hline Data set & AT-PpsA & AT-PpsC & L-AT-Mas & $\begin{array}{l}\text { Methylmalonylated } \\
\text { L-AT-Mas }\end{array}$ & S726F L-AT-Mas & $\begin{array}{l}\text { Methylmalonylated } \\
\text { S726F L-AT-Mas }\end{array}$ & $\begin{array}{l}\text { Malonylated S726F } \\
\text { L-AT-Mas }\end{array}$ & $\begin{array}{l}\text { M624V-S726F L-AT-Mas } \\
\text { soaked in MM-CoA }\end{array}$ \\
\hline PDB code & 7AKC & 7AHB & 7AGP & 7AGS & 7AGQ & 7AGU & 7AGT & 7AGR \\
\hline \multicolumn{8}{|l|}{ Data collection } & SOLEIL PX1 \\
\hline Space group & $P 4_{2} 2_{1} 2$ & $P 1$ & $C 222_{1}$ & $C 222_{1}$ & $C 222_{1}$ & $C 222_{1}$ & $C 222_{1}$ & $C 222_{1}$ \\
\hline $\begin{array}{l}\text { Unit cell } a, b, c(\AA) \\
{\left[\alpha, \beta, \gamma\left({ }^{\circ}\right)\right] \text { for } P 1}\end{array}$ & $94.07,94.07,73.26$ & $\begin{array}{l}51.37,58.36,65.86 \\
{[65.84,73.28,71.02]}\end{array}$ & $\begin{array}{l}146.03,157.14 \\
114.25\end{array}$ & $\begin{array}{l}147.74,156.59 \\
115.52\end{array}$ & $\begin{array}{l}151.42,154.10 \\
115.61\end{array}$ & $148.15,157.97,113.30$ & $\begin{array}{l}148.48,156.12 \\
115.55\end{array}$ & $151.80,152.23,115.13$ \\
\hline Resolution range $(\AA)^{a}$ & $\begin{array}{l}28.9-1.60 \\
(1.69-1.60)\end{array}$ & $\begin{array}{l}48.12-1.90 \\
(1.97-1.90)\end{array}$ & $\begin{array}{l}49.30-2.40 \\
(2.54-2.40)\end{array}$ & $\begin{array}{l}23.49-3.10 \\
(3.27-3.10)\end{array}$ & $\begin{array}{l}48.84-2.80 \\
(2.97-2.80)\end{array}$ & $\begin{array}{l}47.13-3.10 \\
(3.29-3.10)\end{array}$ & $\begin{array}{l}49.11-2.90 \\
(3.08-2.90)\end{array}$ & $\begin{array}{l}48.70-2.80 \\
(2.97-2.80)\end{array}$ \\
\hline No. of unique reflections & $43,463(6,973)$ & $49,339(4,913)$ & $51,153(8,121)$ & $23,423(3,481)$ & $33,365(5,288)$ & $25,082(3,968)$ & $29,200(4,653)$ & $33,129(5,286)$ \\
\hline Completeness (\%) & $98.6(99.7)$ & $96.4(96.1)$ & $99.1(98.4)$ & $95.8(98.1)$ & $99.7(99.5)$ & $99.8(99.3)$ & $97.0(97.2)$ & $99.9(99.7)$ \\
\hline Redundancy & $14.4(14.4)$ & $2.1(2.2)$ & $3.5(3.5)$ & $3.4(3.4)$ & $5.4(5.5)$ & $6.2(6.2)$ & $3.8(3.5)$ & $7.3(7.4)$ \\
\hline$<I / \sigma(I)>$ & $31.0(2.6)$ & $13.7(2.3)$ & $15.8(1.4)$ & $8.6(1.8)$ & $17.1(1.3)$ & $14.3(1.1)$ & $16.3(1.3)$ & $19.0(1.0)$ \\
\hline$R_{\text {merge }}(\%)$ & $4.0(109.2)$ & $5.0(39.7)$ & $4.2(84.5)$ & $8.7(64.4)$ & $6.7(122.0)$ & $9.1(151.1)$ & $6.1(97.1)$ & $6.2(189.1)$ \\
\hline Wilson B factor $\left(\AA^{2}\right)$ & 27.5 & 20.2 & 74.1 & 100.8 & 90.8 & 118.5 & 95.3 & 105.1 \\
\hline $\begin{array}{l}\text { Refinement } \\
\text { No. of reflections } \\
\text { (work / test) }\end{array}$ & $40,599 / 2,126$ & $47,651 / 1,677$ & $48,619 / 2,534$ & $22,200 / 1,188$ & $31,781 / 1,584$ & $23,853 / 1,229$ & $27,778 / 1,422$ & $31,390 / 1,737$ \\
\hline$R_{\text {work }} / R_{\text {free }}(\%)$ & $0.2445 / 0.2671$ & $0.1878 / 0.2240$ & $0.1966 / 0.2193$ & $0.1752 / 0.1951$ & $0.1967 / 0.2307$ & $0.1830 / 0.2083$ & $0.1914 / 0.2091$ & $0.1958 / 0.2187$ \\
\hline No. of molecules/AU ${ }^{b}$ & 1 & 2 & 2 & 2 & 2 & 2 & 2 & 2 \\
\hline Average B factors $\left(\AA^{2}\right)$ & 44.6 & 28.5 & 85.1 & 105.7 & 99.5 & 125.7 & 100.8 & 115.0 \\
\hline \multicolumn{9}{|c|}{ No. of non-hydrogen atoms } \\
\hline Protein & 1,902 & 4,887 & 6,291 & 6,251 & 6,319 & 6,303 & 6,364 & 6,285 \\
\hline Ligands & & 79 & 8 & 7 & 14 & 11 & 12 & 4 \\
\hline Ions & 2 & & & & & & & \\
\hline Water molecules & 195 & 543 & 238 & 29 & 113 & 32 & 102 & 20 \\
\hline \multicolumn{9}{|l|}{ Active site occupation } \\
\hline Chain A & None & None & None & MM-acylation & Acetate & MM-acylation & M-acylation & None \\
\hline Chain B & - & None & Ethylene glycol & None & Acetate & MM-acylation & M-acylation & None \\
\hline \multicolumn{9}{|l|}{ RMS deviations } \\
\hline Bond lengths $(\AA)$ & 0.010 & 0.009 & 0.011 & 0.011 & 0.011 & 0.011 & 0.011 & 0.011 \\
\hline Bond angles $\left({ }^{\circ}\right)$ & 1.08 & 0.820 & 1.06 & 1.06 & 1.07 & 1.09 & 1.08 & 1.07 \\
\hline \multicolumn{9}{|l|}{ Ramachandran plot (\%) } \\
\hline Most favored & 97.3 & 97.5 & 98.5 & 97.4 & 97.5 & 98.3 & 98.3 & 98.4 \\
\hline Allowed / disallowed & $100.0 / 0.0$ & $100.0 / 0.0$ & $100.0 / 0.0$ & $100.0 / 0.0$ & $99.9 / 0.1$ & $99.9 / 0.1$ & $100.0 / 0.0$ & $99.9 / 0.1$ \\
\hline
\end{tabular}

${ }^{b}$ Asymmetric unit. 
Supporting Information Table 2. PDB AT structures that include a L linker domain.

\begin{tabular}{|c|c|c|c|c|c|c|c|c|c|}
\hline $\begin{array}{l}\text { PDB code- } \\
\text { chain }\end{array}$ & Protein name & Domain organization $^{b}$ & Extender unit & $\mathbf{Z}$ & $\operatorname{Rmsd}(\AA)$ & $\begin{array}{l}\text { Nb. residues } \\
\text { aligned }\end{array}$ & $\begin{array}{c}\text { Nb. } \\
\text { residues }\end{array}$ & $\begin{array}{l}\% \\
\text { Id }\end{array}$ & $\begin{array}{l}\text { Rotation } \\
\text { angle }\left({ }^{\circ}\right)^{c}\end{array}$ \\
\hline $7 \mathrm{AGP}^{-\mathrm{A}^{a}}$ & Mas & KS-L-AT-DH-ER-KR-ACP & MM-CoA & - & - & - & - & - & $0^{(1)}$ \\
\hline 6FIJ-B & Cercosporin PKS CTB1 & $\begin{array}{l}\text { SAT-KS-L-MAT-PT-ACP1-ACP2- } \\
\text { TE }\end{array}$ & $\mathrm{M}-\mathrm{CoA}$ & 12.6 & 1.5 & 77 & 1275 & 21 & $56^{(2)}$ \\
\hline 5BP1-A & Mas-like PKS & KS-L-AT-DH- $\Psi$ KR-ER-KR-ACP & MM-CoA & 12.4 & 1.4 & 76 & 817 & 47 & $9^{(1)}$ \\
\hline 6FIK-B & Cercosporin PKS CTB1 & $\begin{array}{l}\text { SAT-KS-L-MAT-PT-ACP1-ACP2- } \\
\text { TE crosslinked to ACP2 }\end{array}$ & $\mathrm{M}-\mathrm{CoA}$ & 12.4 & 1.5 & 77 & 1275 & 21 & $59^{(2)}$ \\
\hline 2QO3B-B & DEBS module 3 & KS-L-AT-KR ${ }^{0}$-ACP & MM-CoA & 11.8 & 1.4 & 73 & 873 & 38 & $25^{(3)}$ \\
\hline 6C9U-A & DEBS module 3 & $\begin{array}{l}\text { KS-L-AT-KR }{ }^{0} \text {-ACP } \\
+ \text { TE, Fab-bound complex }\end{array}$ & $\mathrm{MM}-\mathrm{CoA}$ & 11.8 & 1.3 & 72 & 897 & 38 & $28^{(3)}$ \\
\hline 6J0U-C & Ansamitocin PKS module 3 & KS-L-AT-DH-KR-ACP & MOM-ACP & 11.7 & 1.7 & 74 & 419 & 32 & $36^{(4)}$ \\
\hline 2HG4-C & DEBS module 5 & KS-L-AT-KR-ACP & MM & 11.6 & 1.3 & 72 & 882 & 33 & $31^{(4)}$ \\
\hline 3TZX-B & Pks13 & ACP1-KS-L-AT-ACP2-TE & $\mathrm{C}_{24-26-c a r b o x y a c y l-C o A}$ & 11.3 & 1.5 & 74 & 467 & 26 & 57 \\
\hline 5YDA-A & Splenocin PKS SpnD & KS-L-AT-ACP-TE & $\begin{array}{l}\text { Broadly selective } \\
\text { CoA substrates }\end{array}$ & 11.2 & 1.6 & 74 & 409 & 32 & $30^{(3)}$ \\
\hline 6IYR-A & Salinomycin PKS module 8 & KS-L-AT-DH-ER-KR-ACP & MM-CoA & 10.8 & 1.5 & 73 & 423 & 33 & $24^{(3)}$ \\
\hline 6IYT-A & Salinomycin PKS module 14 & KS-L-AT-KR-ACP & EM-CoA & 10.1 & 1.1 & 67 & 432 & 27 & $26^{(3)}$ \\
\hline 4MZ0-B & Curacin A PKS module CurL & KS-L-AT-OM/MT-KR-ACP & M-CoA & 9.4 & 1.3 & 72 & 823 & 32 & $39^{(5)}$ \\
\hline 4QBU-A & Zwittermicin A PKS ZmaA & KS-KR-ACP1-KS-L-AT-KS-ACP2-C & HM-ACP & 9.3 & 1.8 & 72 & 436 & 29 & $33^{(5)}$ \\
\hline 4AMM-A & Enediyne PKS DynE8 & KS-L-AT-ACP-KR-DH-PPTase & M-CoA & 9 & 2.1 & 75 & 384 & 19 & 55 \\
\hline 6IYO-A & Salinomycin PKS module 2 & KS-L-AT-DH-ER-KR-ACP & M-CoA & 5 & 1.2 & 44 & 421 & 30 & $34^{(5)}$ \\
\hline 2JFD-D & Human FAS & $\begin{array}{l}\text { KS-L-MAT-DH- } \Psi M E-\Psi K R-E R-K R- \\
\text { ACP-TE }\end{array}$ & $\begin{array}{l}\text { Acetyl-CoA } \\
\text { M-CoA }\end{array}$ & 2.6 & 3.1 & 51 & 399 & 22 & $37^{(5)}$ \\
\hline
\end{tabular}

${ }^{a}$ This work.

${ }^{b}$ The fragments used for structure determination are in bold. Abbreviations: A, acetyl; AT, acyltransferase; B, branching domain; C, condensation domain; DEBS, 6deoxyerythronolide B synthase; EM, ethylmalonyl; HM, Hydroxymalonyl; KR ${ }^{0}$, redox-inactive ketoreductase domain ; L, linker domain; M, malonyl; MAT, malonyl-CoAspecific acyltransferase; MM, methylmalonyl; MOM, Methoxymalonyl; NR, nonreducing; OM, O-methyltransferase; PPTase, phosphopantetheinyl transferase ; PT, product template domain; SAT, starter unit acyltransferase.

${ }^{c}$ Rotation angle required to superpose the L domains once having superimposed the AT domains using the structure of L-AT-Mas as a reference. Structures sharing the same numbers (given in superscript and in parentheses) share the same orientation of their L domain. 


\section{SUPPORTING INFORMATION REFERENCES}

1. Chalut, C., Botella, L., de Sousa-D'Auria, C., Houssin, C., and Guilhot, C. (2006) The nonredundant roles of two 4'-phosphopantetheinyl transferases in vital processes of Mycobacteria, Proc. Natl. Acad. Sci. U S A 103, 8511-8516.

2. Massemin, A., Cabantous, S., Waldo, G. S., and Pedelacq, J. D. (2019) High-throughput isolation of soluble protein domains using a bipartite split-GFP complementation system, Methods Mol. Biol. 2025, 321-333.

3. Pedelacq, J. D., Nguyen, H. B., Cabantous, S., Mark, B. L., Listwan, P., Bell, C., Friedland, N., Lockard, M., Faille, A., Mourey, L., Terwilliger, T. C., and Waldo, G. S. (2011) Experimental mapping of soluble protein domains using a hierarchical approach, Nucleic Acids Res. 39, e125.

4. Cabantous, S., Terwilliger, T. C., and Waldo, G. S. (2005) Protein tagging and detection with engineered self-assembling fragments of green fluorescent protein, Nat. Biotechnol. 23, 102-107.

5. Pedelacq, J. D., Waldo, G. S., and Cabantous, S. (2019) High-throughput protein-protein interaction assays using tripartite split-GFP Complementation, Methods Mol. Biol. 2025, 423-437.

6. Gavalda, S., Leger, M., van der Rest, B., Stella, A., Bardou, F., Montrozier, H., Chalut, C., Burlet-Schiltz, O., Marrakchi, H., Daffe, M., and Quemard, A. (2009) The Pks13/FadD32 crosstalk for the biosynthesis of mycolic acids in Mycobacterium tuberculosis, J. Biol. Chem. 284, 19255-19264.

7. Franke, D., Petoukhov, M. V., Konarev, P. V., Panjkovich, A., Tuukkanen, A., Mertens, H. D. T., Kikhney, A. G., Hajizadeh, N. R., Franklin, J. M., Jeffries, C. M., and Svergun, D. I. (2017) ATSAS 2.8: a comprehensive data analysis suite for small-angle scattering from macromolecular solutions, J. Appl. Crystallogr. 50, 1212-1225.

8. Kabsch, W. (2010) XDS, Acta Crystallogr. D Biol. Crystallogr. 66, 125-132.

9. Collaborative Computational Project, N. (1994) The CCP4 suite: programs for protein crystallography, Acta Crystallogr. D Biol. Crystallogr. 50, 760-763.

10. Murshudov, G. N., Skubak, P., Lebedev, A. A., Pannu, N. S., Steiner, R. A., Nicholls, R. A., Winn, M. D., Long, F., and Vagin, A. A. (2011) REFMAC5 for the refinement of macromolecular crystal structures, Acta Crystallogr. D Biol. Crystallogr. 67, 355-367.

11. McCoy, A. J., Grosse-Kunstleve, R. W., Adams, P. D., Winn, M. D., Storoni, L. C., and Read, R. J. (2007) Phaser crystallographic software, J. Appl. Crystallogr. 40, 658-674.

12. Emsley, P., Lohkamp, B., Scott, W. G., and Cowtan, K. (2010) Features and development of Coot, Acta Crystallogr. D Biol. Crystallogr. 66, 486-501.

13. Afonine, P. V., Grosse-Kunstleve, R. W., Chen, V. B., Headd, J. J., Moriarty, N. W., Richardson, J. S., Richardson, D. C., Urzhumtsev, A., Zwart, P. H., and Adams, P. D. (2010) phenix.model_vs_data: a high-level tool for the calculation of crystallographic model and data statistics, J. Appl. Crystallogr. 43, 669-676.

14. Bricogne, G., Blanc, E., Brandl, M., Flensburg, C., Keller, P., Paciorek, W., Roversi, P., Sharff, A., Smart, O. S., Vonrhein, C., and Womack, T. O. (2017) BUSTER, 2.10.3 ed., Cambridge, United Kingdom: Global Phasing Ltd.

15. Holm, L., and Park, J. (2000) DaliLite workbench for protein structure comparison, Bioinformatics 16, 566-567.

16. Holm, L., and Rosenstrom, P. (2010) Dali server: conservation mapping in 3D, Nucleic Acids Res. 38, W545-549.

17. Kabsch, W., and Sander, C. (1983) Dictionary of protein secondary structure: pattern recognition of hydrogen-bonded and geometrical features, Biopolymers 22, 2577-2637. 
18. Touw, W. G., Baakman, C., Black, J., te Beek, T. A., Krieger, E., Joosten, R. P., and Vriend, G. (2015) A series of PDB-related databanks for everyday needs, Nucleic Acids Res. 43, D364-368.

19. DeLano, W. L. (2002) The PyMOL Molecular Graphics System, Schrödinger, LCC, New York.

20. Robert, X., and Gouet, P. (2014) Deciphering key features in protein structures with the new ENDscript server, Nucleic Acids Res. 42, W320-324.

21. Marrakchi, H., Laneelle, M. A., and Daffe, M. (2014) Mycolic acids: structures, biosynthesis, and beyond, Chem. Biol; 21, 67-85.

22. Afonine, P. V., Moriarty, N. W., Mustyakimov, M., Sobolev, O. V., Terwilliger, T. C., Turk, D., Urzhumtsev, A., and Adams, P. D. (2015) FEM: feature-enhanced map, Acta Crystallogr. D Biol. Crystallogr. 71, 646-666. 
a

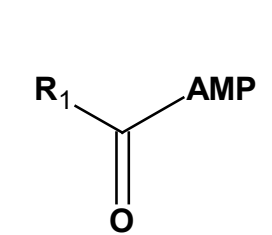

$\prod_{O}^{\mathrm{O}_{\mathrm{CoA}}} \underset{\text { Trehalose (Tre) }}{\stackrel{\mathrm{Pks13}}{\mathrm{R}}}$<smiles>[R]C(=O)C([R2])C(=O)O[Tl]</smiles>

b<smiles>[R]C(=O)O</smiles>

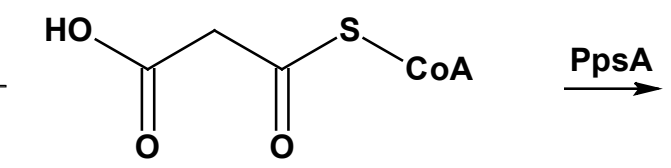

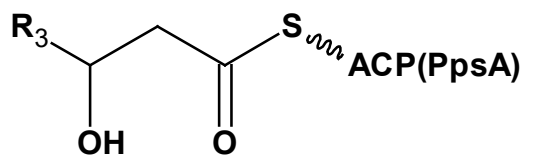<smiles></smiles><smiles>CCCCCC#CSC(=O)CC(=O)O</smiles><smiles>[R5]C(O)CC(O)CCCC(=O)S[14C]([14CH])=O</smiles>

C<smiles>[R]C(=O)SC(=O)O</smiles>

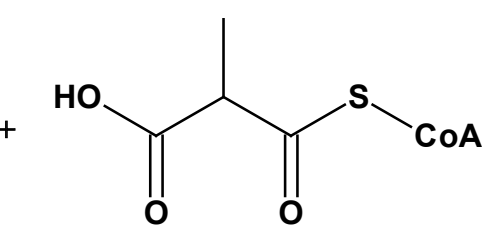

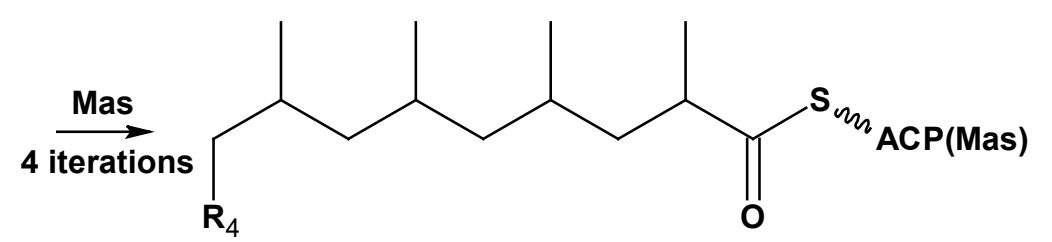

Supplementary Information Figure 1 
a

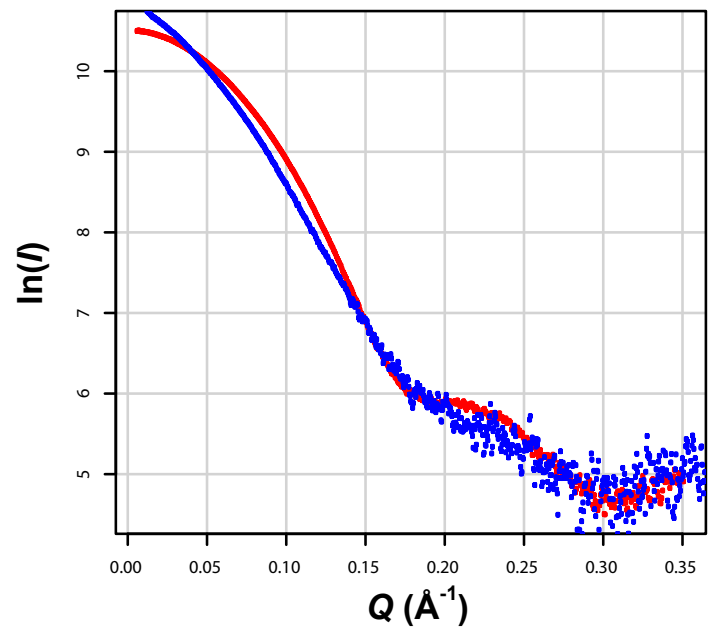

AT-Mas (542-959)

AT-Mas (621-952)

b

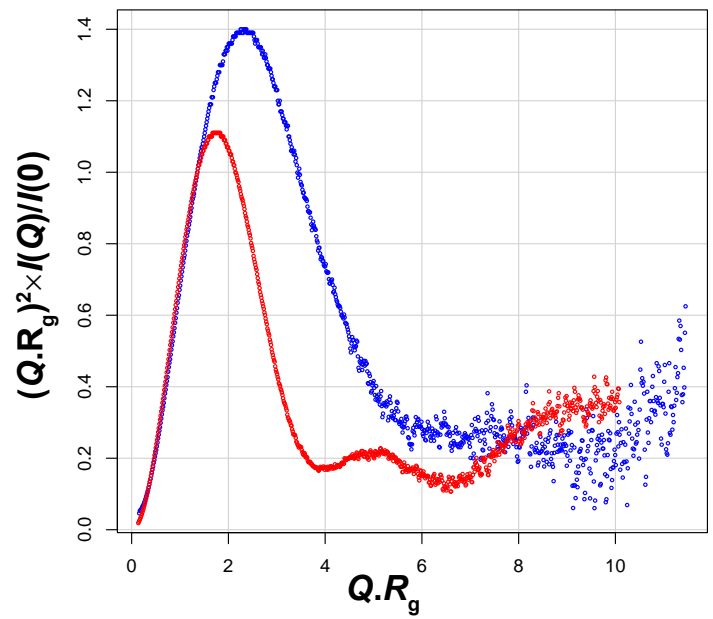

AT-Mas (542-959) AT-Mas (621-952)

Supplementary Information Figure 2 
L-AT-Pks 13 L-AT-Pks 13 L-AT-Mas AT-PpsA
AT-PpsC

L-AT-MaS AT-PPSA $A T-P p S C$

L-AT-Pks 13 L-AT-Pks 13 L-AT-Mas AT-PpsA
AT-PpsC

L-AT-Mas
AT-PpsA AT-PPSC

L-AT-Pks 13 L-AT-Pks 13 L-AT-Mas AT-PpsA AT-PpsC

L-AT-MaS $A T-P P s A$
$A T-P P S C$

L-AT-Pks 13 L-AT-Pks 13 L-AT-Mas AT-PpsA

L-AT-MaS AT-PPsA $A T-P P s A$
$A T-P P s C$

L-AT-Pks 13 L-AT-Pks 13 L-AT-Mas AT-PpsA
AT-PpsC

L-AT-Mas AT-PPsA AT-PPsC

L-AT-Pks 13 L-AT-Pks 13 L-AT-Mas AT-PpsA AT-PpsC

L-AT-Mas AT-PPsA
AT-PPsC

L-AT-Pks 13 L-AT-Pks 13 L-AT-Mas AT-PpsA AT-PpsC

L-AT-Mas $A T-P p s A$
$A T-P p s C$

L-AT-Pks 13

L-AT-Pks 13 L-AT-Mas AT-PpsA

L-AT-MaS AT-PpsA $A T-P p s A$
$A T-P p s C$

$\alpha 1$

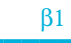

$\alpha 2$

576 RFDEF GNIT DSAVAEEPEPELPGVTEEALRLKEAALEELAAQEVTAPLVPLAVSAFLTSRKKAAAAELA 447 $\ldots \ldots \ldots \ldots \ldots \ldots \ldots \ldots \ldots \ldots \ldots \ldots$ PRLFMLSSTSSDALRQTARQLA .

lele. elee lelelele

$\stackrel{\beta 2}{\longrightarrow}$. elelelelelel

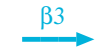

DWMOSPEGOASSIFSIGRSIS.RRNHGRSRAVVIAHDHDEATKGI RAVAAG KOAPNVFSV ....DGPVT 469 TWVEE . HOD CVAASDLAYTLATGRAHRPVRTAVVAANLPELVEGLREVADG da lyda avg . . . . . HGD $621 \ldots \ldots \ldots \ldots \ldots \ldots \ldots \ldots \ldots \ldots \ldots \ldots \ldots \ldots$ 546
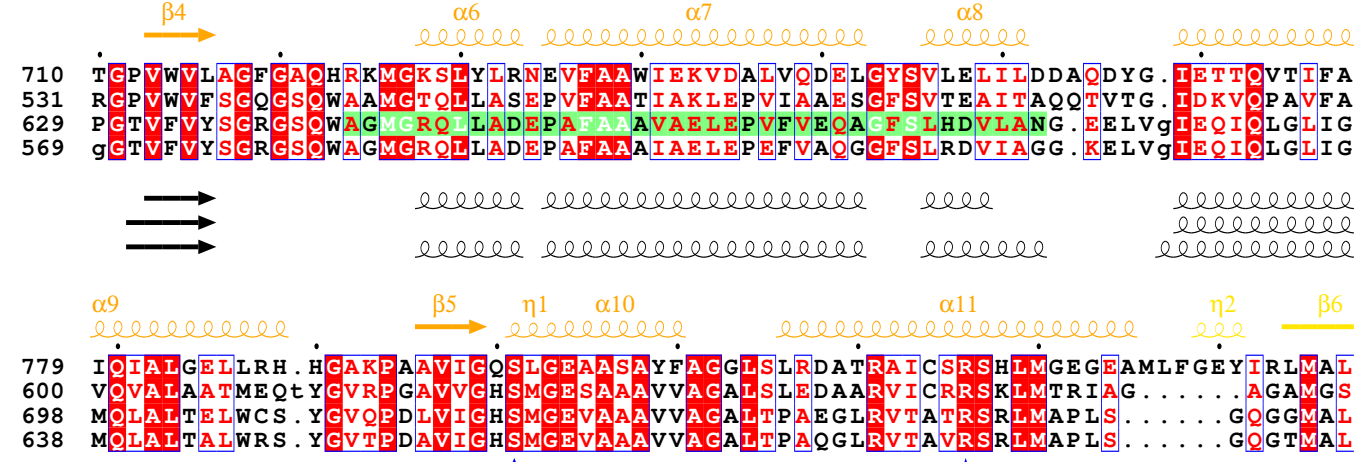
638 MOLALTALWRS. YGVTPDAVIGHSMG EVAAAVVAGA T PAOGLRVTAVRSRLMAPLS.......GOGTMAI

eleecelee beblebel rebelelel

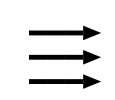

- beceloce elebelele

leberebêlece cheleleleleleres belecelelelebel

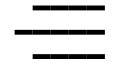

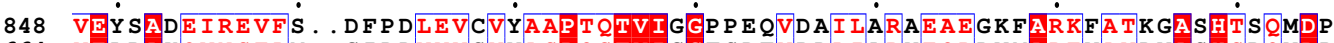

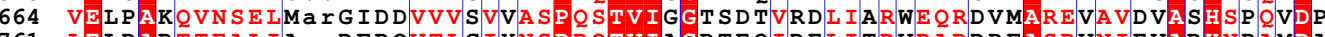
761 LELDAPTTEALIA. DFPQVTLGIYNSPRQTVIAGP TEQIDELITRVRARDRFASRVNIEVAPHNPAMDA 701 LELDAEATEALIA. DYP EVSLGIYASPRQTVISGPPLLIDELIDKVRQONGFATRVNIEVAPHNP AMDA

- elevelee

- elelelel
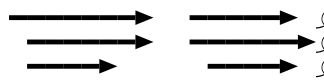

clebelel relelelelel

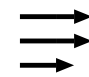

elee $\alpha 14$

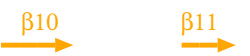

elee

\section{eleepel}

916

734
829

829
769

LLGE TAEIQGIKPT SPTCGIFST் HE . GRYIKP

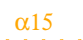

$\alpha 16$ T

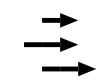

$\stackrel{\beta 12}{\longrightarrow}$

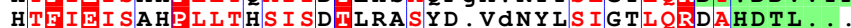

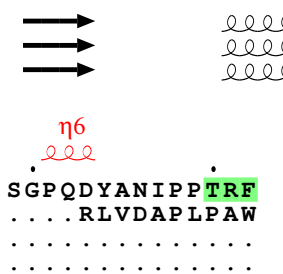


a
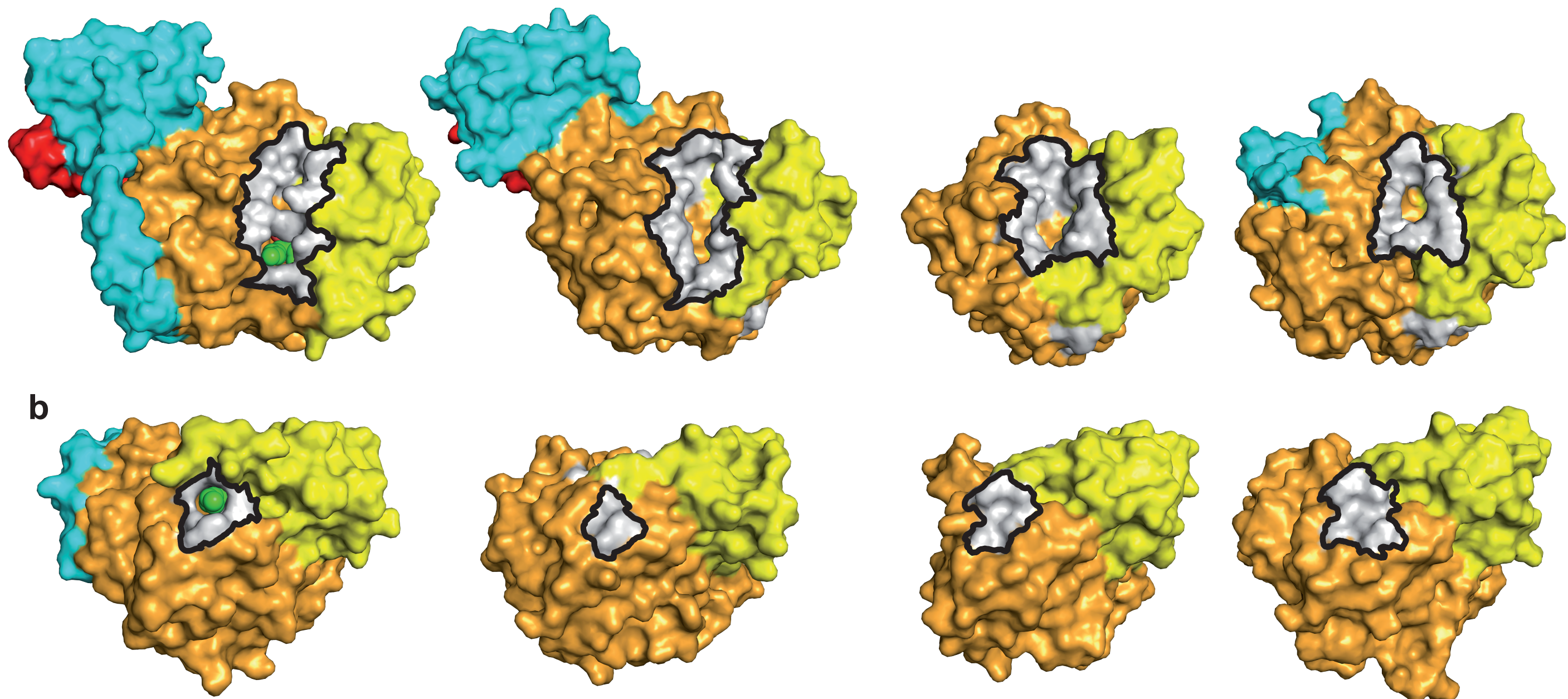

Carboxypalmitoylated L-AT-Pks13

L-AT-Mas

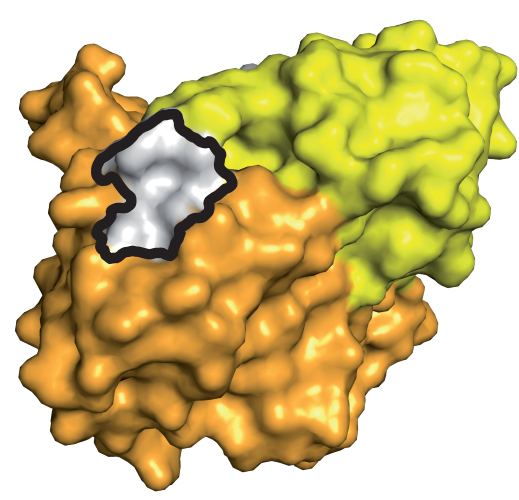

AT-PpsA

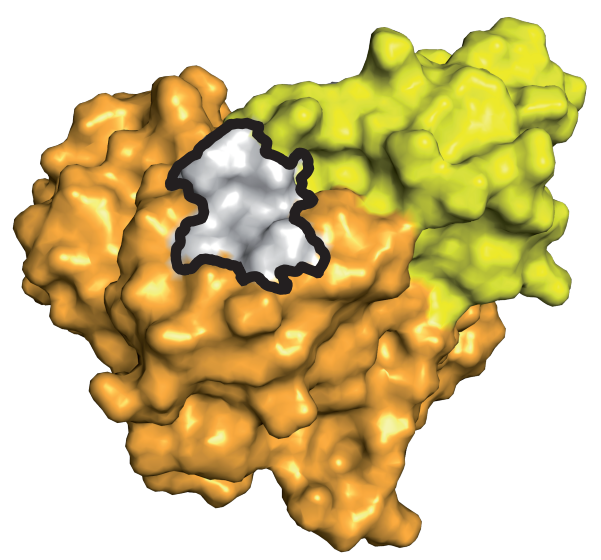

AT-PpsC

Supplementary Information Figure 4 
a

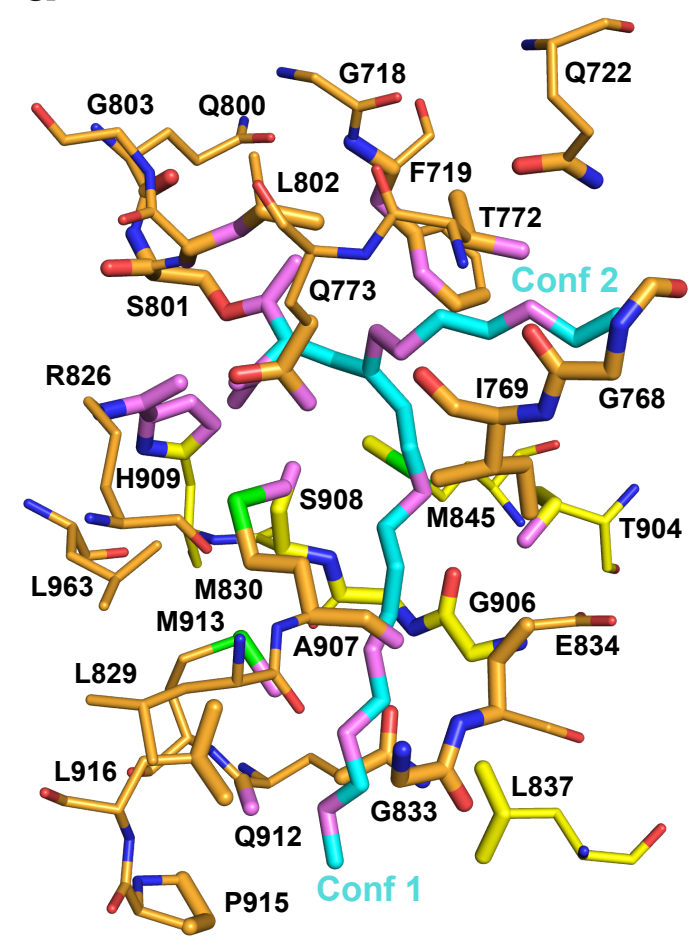

Carboxypalmitoylated L-AT-Pks13 b

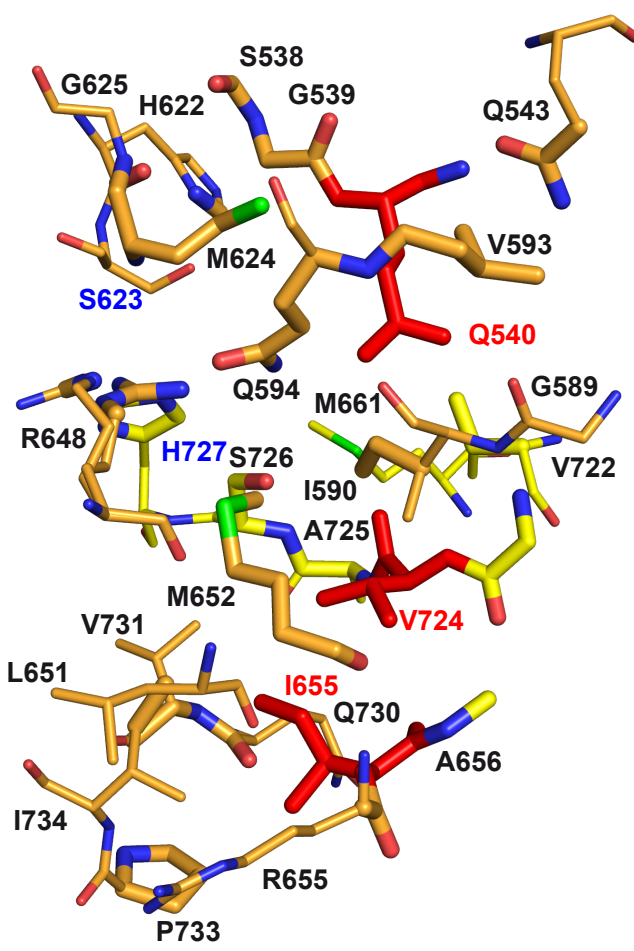

C

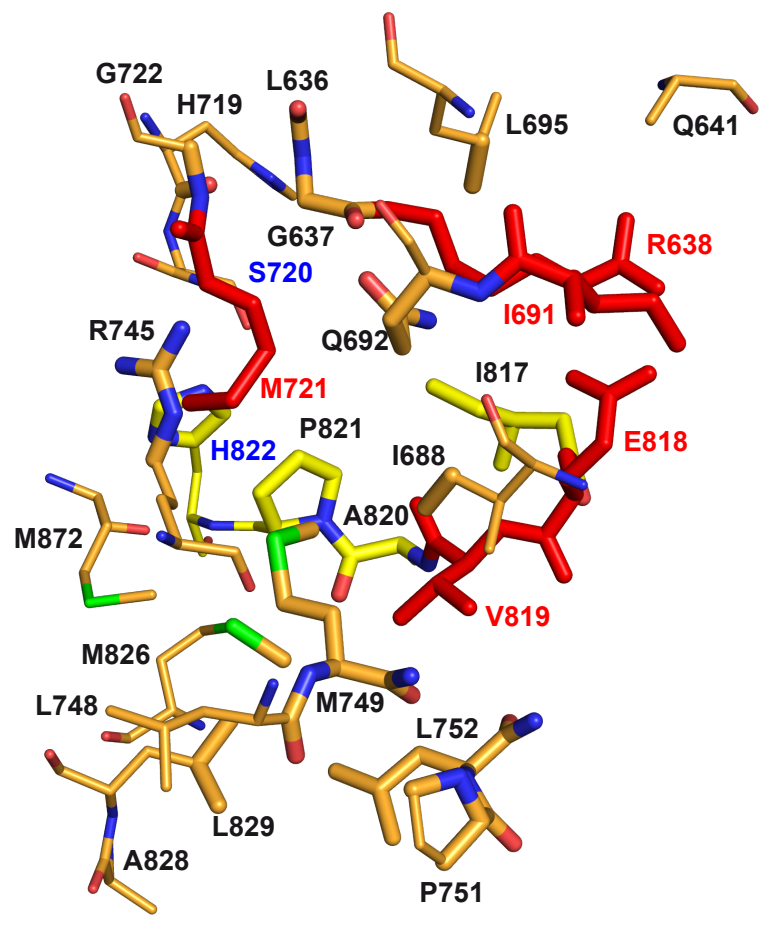

d

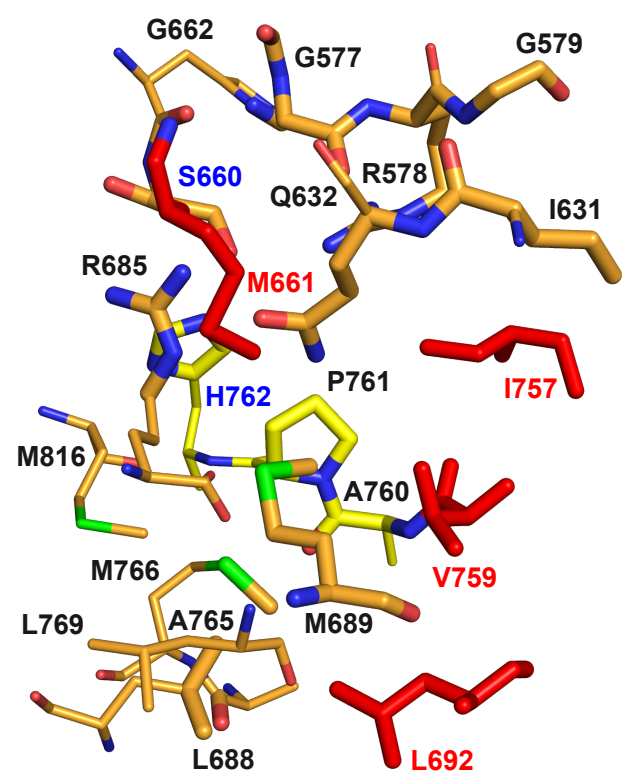

Supplementary Information Figure 5

AT-PpsA

AT-PpsC 
